isid/ms/2011/04

July 20, 2011

http://www.isid.ac.in/statmath/eprints

\title{
Involutions on the second duals of group algebras versus subamenable groups
}

\author{
AJit IQBAl Singh
}

Indian Statistical Institute, Delhi Centre 7, SJSS Marg, New Delhi-110 016, India 



\title{
INVOLUTIONS ON THE SECOND DUALS OF GROUP ALGEBRAS VERSUS SUBAMENABLE GROUPS
}

\author{
AJIT IQBAL SINGH
}

\begin{abstract}
Let $L^{1}(G)^{* *}$ be the second dual of the group algebra $L^{1}(G)$ of a locally compact group $G$. We study the question of involutions on $L^{1}(G)^{* *}$. A new class of subamenable groups is introduced which is universal for all groups. There is no involution on $L^{1}(G)^{* *}$ for a subamenable group $G$.
\end{abstract}

\section{INTRODUCTION}

Let $A$ be a complex Banach algebra, $A^{*}$ its dual and $A^{* *}$ its second dual. We follow R. Arens ([A1], [A2]) and equip $A^{* *}$ with the first Arens product $\square$ or the second Arens product $\diamond$ defined as follows.

For $\varphi, \psi \in A, f \in A^{*}, F$ and $H \in A^{* *}$,

$$
f \varphi(\psi)=f(\varphi \psi), \quad F f(\varphi)=F(f \varphi), \quad H \square F(f)=H(F f) ;
$$

whereas $\varphi f(\psi)=f(\psi \varphi), f F(\varphi)=F(\varphi f), F \diamond H(f)=H(f F)$. The Banach algebra $A$ is said to be Arens regular if $\square=\diamond$.

For a continuous conjugate linear map $T: A \rightarrow A$, the conjugate-adjoint $T^{c}: A^{*} \rightarrow A^{*}$ and the second conjugate-adjoint $T^{c c}: A^{* *} \rightarrow A^{* *}$ are defined via

$$
T^{c} f=\overline{f \circ T}, \quad T^{c c} F=\overline{F \circ T^{c}} \text { for } f \in A^{*}, F \in A^{* *} .
$$

Both $T^{c}$ and $T^{c c}$ are conjugate linear mappings and can very well be called the first adjoint and the second adjoint of $T$ respectively. We will denote them by $T^{*}$ and $T^{* *}$ respectively only if no confusion can arise.

P. Civin and B. Yood ([CY]) noted that a continuous (algebra) involution on $A$, i.e. a continuous conjugate linear anti-homomorphism of period two, can be extended to an involution on $A^{* *}$ if $A$ is Arens regular. The converse is also true. On the other hand, M. Grosser [G] showed that a necessary condition for the bidual $A^{* *}$ of $A$ with bounded approximate right identity to admit an involution with respect to the first Arens product

2010 Mathematics Subject Classification. Primary 43A20, 43A22; Secondary 46K99, 54D35.

Key words and phrases. Arens product, group algebra, involutions, second dual, Stone-Čech compactification, subamenable group. 
is that $A^{*} A=A^{*}$. To get an idea for the case of $A=L^{1}(G)$, the group algebra of a locally compact (Hausdorff) group (with left Haar measure), we just mention that (i) $A$ is Arens regular if and only if $G$ is finite, as proved by Civin and Yood [CY] for the abelian case and by N. Young [Y] for the general case; and (ii) $A^{*} A=A^{*}$ if and only if $G$ is discrete as indicated by Civin and Yood for the abelian case in the proof of Theorem 3.12 [CY] and proved by Grosser $[\mathrm{G}]$ for the general case. We may record the important consequence due to Grosser.

Theorem 1.1. [G] If $L^{1}(G)^{* *}$ admits an involution then $G$ is discrete.

The background for $L^{1}(G)^{* *}$ for discrete $G$ is well set by M.M. Day [Da] and Civin and Yood [CY] and an important role is played by the Stone-Čech compactification $\beta G$ of $G$ identified as a semigroup under the multiplication $\square$ of $L^{1}(G)^{* *}$. See also R.G. Douglas [Do].

The question of involutions on the second duals of group algebras was initially given attention by J. Duncan and S.A.R. Hosseiniun $[\mathrm{DH}]$ and further studied by H. Farhadi and F. Ghahramani [FG]; see also the related paper by M. Neufang [N].

Our interest in this short paper is to further strengthen Theorem 1.1 above. We confine our attention to the first Arens product only. We define the notion of subamenability for a locally compact (Hausdorff) group $G$ and prove

Theorem 1.2. Let $G$ be a subamenable non-compact locally compact group. Then $L^{1}(G)^{* *}$ has no involution.

We also show that the class of subamenable groups properly contains that of amenable groups. Another interesting fact is that every discrete group $G$ is a subgroup of a subamenable discrete group $\widetilde{G}$ with cardinality $\# \widetilde{G} \leq 2^{\# G}$.

We may refer to E. Hewitt and K. A. Ross [HR], J.-P. Pier [Pi], T. W. Palmer [Pal], N. Hindman $[\mathrm{H}]$ and N. Hindman and D. Strauss ([HS1], [HS3]), M. Filali and A.I. Singh [FSi] and H.G. Dales, A.T.-M. Lau and D. Strauss [DLS], for instance, for basics and some further details. These sources permit us to concentrate on our new results utilizing the relevant formulations of known results rather than tracing their history.

\section{Subamenable groups And the question of involutions on $L^{1}(G)^{* *}$}

We begin with a bit of prevalent notation and a variant of the notion of amenability for a locally compact (Hausdorff) group $G$ with identity $e$ and left Haar measure $\lambda$, simply denoted by $d x$ at times. 
Let $k(G)$ be the minimal number of compact sets required to cover $G$. Clearly, $k(G)$ is an infinite cardinal number except when $G$ is compact, and in that case $k(G)=1$. Further, for an infinite discrete group, $k(G)=k$, the cardinality $\# G$ of $G$.

For $x \in G$ and a function $f$ on $G$, let $\ell_{x} f$ be the function on $G$ given by $\ell_{x} f(y)=f(x y)$ for $y$ in $G$. For $F$ in $L^{1}(G)^{* *}=L^{\infty}(G)^{*}$ let $L_{F}$ be the bounded linear operator on $L^{\infty}(G)$ to itself given by $L_{F} f=F f$. Let 1 be the constant function one on $G$. A mean $M$ on $G$ is a positive linear functional $M$ on $L^{\infty}(G)$ with $M(\mathbf{1})=1$. In case $G$ is discrete, $L^{1}(G)$, $L^{\infty}(G), L^{1}(G)^{* *}$ etc. will be denoted, at times, by $\ell^{1}(G), \ell^{\infty}(G), \ell^{1}(G)^{* *}$ etc. respectively. For a subset $W$ of a vector space $X, \Gamma(W)$ will denote its linear span.

Definition 2.1. (i) A non-empty subset $\mathcal{F}$ of $L^{1}(G)^{* *}$ will be called an invoid for $G$ if $\Gamma(\mathcal{F})$ is a left ideal in $L^{1}(G)^{* *}$.

(ii) An invoid for $G$ containing at least one mean $M$ will be called a meanoid for $G$.

(iii) For a cardinal number $\alpha, G$ will be called $\alpha$-amenable if $G$ has a meanoid with cardinality $\leq \alpha$.

(iv) $G$ will be called subamenable if $G$ is $\alpha$-amenable for some $1 \leq \alpha<2^{2^{k(G)}}$, i.e., $L^{1}(G)^{* *}$ possesses a left ideal which has dimension $<2^{2^{k(G)}}$ and contains a mean.

The definition is designed to have the following result.

Theorem 2.2. Let $G$ be a subamenable non-compact locally compact group. Then $L^{1}(G)^{* *}$ has no involution.

Proof. By definition of subamenability, $L^{1}(G)^{* *}$ has a $\gamma$-dimensional left ideal with $1 \leq$ $\gamma<2^{2^{k(G)}}$. By Filali and P. Salmi ([FSa, Theorem 6]), $L^{1}(G)^{* *}$ has no non-trivial right ideal with dimension $<2^{2^{k(G)}}$. So $L^{1}(G)^{* *}$ has no involution.

We now come to the reason for nomenclature in Definition 2.1.

Remark 2.3. Let $\mathcal{F}$ be a nonempty subset of $L^{1}(G)^{* *}$.

(i) The set $\mathcal{F}$ is an invoid for $G$ if and only if for each $F$ in $\mathcal{F}$ and $H$ in $L^{1}(G)^{* *}$ there exist $F_{j} \in \mathcal{F}, h_{j} \in L^{\infty}(G), 1 \leq j \leq n$ satisfying $H_{0}\left(L_{F}-\sum_{j=1}^{n} F_{j} \otimes h_{j}\right)=0$, i.e., for each $f$ in $L^{\infty}(G), H(F f)=\sum_{j=1}^{n} H\left(h_{j}\right) F_{j}(f)$. To see this, we have only to note that $H \square F(f)=H(F f)$ and for a scalar $\beta$ there exist $h \in L^{\infty}(G)$ with $\beta=H(h)$. It is as if for each closed hyperplane $\Pi$ in $L^{\infty}(G)$ there is a finite rank bounded operator $T_{\Pi}$ in $\Gamma(\mathcal{F}) \otimes L^{\infty}(G)$ on $L^{\infty}(G)$ to itself with the range of $L_{F}-T_{\Pi}$ in $\Pi$. 
(ii) For a mean $M$ on $G, \mathcal{F}=\{M\}$ is a meanoid for $G$ if and only if $L_{M}=M \otimes \mathbf{1}$, i.e., $M f=M(f) \mathbf{1}$ for each $f$ in $L^{\infty}(G)$, i.e. $M$ is topologically left invariant. To see this, let $H \in L^{1}(G)^{* *}=L^{\infty}(G)^{*}$ be arbitrary. We note that $H \square M=\beta_{H} M$ for a scalar $\beta_{H}$ if and only if $H(M f)=\beta_{H} M(f)$ for each $f$ in $L^{\infty}(G)$. The basic fact that $M \mathbf{1}=\mathbf{1}$ gives that this is so only if $\beta_{H}=H(\mathbf{1})$. Thus, $H \square M=\beta_{H} M$ for a scalar $\beta_{H}$ if and only if $H(M f)=H(\mathbf{1}) M(f)$ for each $f$ in $L^{\infty}(G)$, i.e., $H_{0}\left(L_{M}-M \otimes \mathbf{1}\right)=0$.

(iii) By (ii) above $G$ is 1-amenable if and only if $G$ is amenable.

(iv) If $G$ is $\alpha_{1}$-amenable and $\alpha_{1}<\alpha_{2}$, then $G$ is $\alpha_{2}$-amenable.

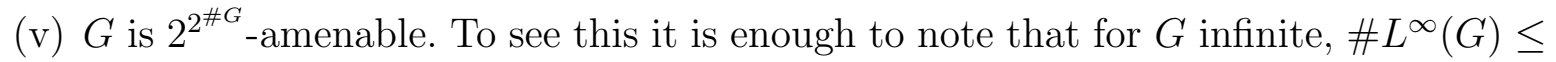
$2^{\# G}$ and, therefore, $\# L^{1}(G)^{* *} \leq 2^{2^{\# G}}$.

(vi) We do not yet have any example of a non-subamenable group. A fortiori, we do not have an example of an infinite $G$ with an involution on $L^{1}(G)^{* *}$. But Theorem 2.9 below gives a large class of subamenable groups.

Corollary 2.4. Let $G$ be an amenable non-compact locally compact group. Then $L^{1}(G)^{* *}$ has no involution.

Proposition 2.5. If $G$ is $m$-amenable for some $m \in \mathbb{N}$, the set of natural numbers, then $G$ is amenable.

Proof. Let $\mathcal{F}$ be a meanoid for $G$ with cardinality $m$. Then $\mathcal{F}$ contains a mean $M$. Since $M \square M(\mathbf{1})=1, M$ is not a right annihilator of $L^{1}(G)^{* *}$.

So $\Gamma(\mathcal{F})$ is a finite-dimensional left-ideal in $L^{1}(G)^{* *}$, which is not generated by right annihilators of $L^{1}(G)^{* *}$. By Filali ([F2, Corollary (3) of Theorem 3]) $G$ is amenable.

We now prepare to show that there exist subamenable non-amenable groups. In fact, we will show that each discrete group $G$ is a subgroup of some subamenable group $G$ with $k\left(G_{\sigma}\right)=\# G_{\sigma} \leq 2^{\# G}$. It is as if the class of subamenable group is universal for all groups.

Remark 2.6. In view of Grosser's Theorem 1.1 in the first section, we can confine our attention to discrete groups. From now onwards $G$ is an infinite discrete group with cardinality $k$ unless otherwise stated.

(i) The result on the dimension of non-trivial right ideals in $L^{1}(G)^{* *}$ by Filali and Salmi used in the proof of Theorem 2.2 is needed only for discrete groups. In that case it also follows from Filali and J. Pym ([FP, Theorem 5]) simply because for discrete $G, \mathcal{L U C}(G)^{*}=L^{1}(G)^{* *}$. 
(ii) An independent proof of Corollary 2.4 can be given using the relevant part and version of an earlier result of Filali ([F1, Theorem 3]) viz., for an infinite discrete group $G, L^{1}(G)^{* *}$ has no non-trivial finite-dimensional right ideal together with the basic fact that for amenable $G, L^{1}(G)^{* *}$ has non-trivial finite dimensional left ideals.

Proposition 2.7. Let $G$ be a discrete group and $\alpha$, a cardinal number.

(i) If $\pi$ is a homomorphism of $G$ onto a discrete group $S$ and $G$ is $\alpha$-amenable then $S$ is $\alpha$-amenable. Equivalently, the quotient group $S$ of $G$ by a normal subgroup $N$ is $\alpha$-amenable if $G$ is so.

(ii) If $S$ is a subgroup of $G$ and $G$ is $\alpha$-amenable then so is $S$.

(iii) If $N$ is an amenable normal subgroup of $G$ with the quotient $G / N$ $\alpha$-amenable then $G$ is $\alpha$-amenable.

Proof. Some basic ingredients for the proof are taken from the well-known amenable version. We may refer to Pier $([\mathrm{Pi}, \S 13])$, for instance.

(i) Let $\mathcal{F}$ be a meanoid for $G$ with $\# \mathcal{F} \leq \alpha$ and $M$ a mean present in $\mathcal{F}$.

For $\xi \in \ell^{\infty}(S)$, let $\hat{\xi}=\xi_{0} \pi$. Then $\hat{\xi} \in \ell^{\infty}(G)$ and is constant on $\pi^{-1}\{b\}$ for each $b$ in $S$.

We put $\ell_{\pi}^{\infty}(G)=\left\{f \in \ell^{\infty}(G): f\right.$ is constant on $\pi^{-1}\{b\}$ for each $b$ in $\left.S\right\}=\{\hat{\xi}: \xi \in$ $\left.\ell^{\infty}(S)\right\}=\widehat{\ell^{\infty}(S)}$. In fact, $\xi \rightarrow \hat{\xi}$ is a 1-1 correspondence between $\ell^{\infty}(S)$ and $\ell_{\pi}^{\infty}(G)$. For $\Phi \in \ell^{1}(S)^{* *}=\ell^{\infty}(S)^{*}$, let $\hat{\Phi}$ be any Hahn-Banach extension to $\ell^{\infty}(G)$ of $\Phi_{1}$ on $\ell_{\pi}^{\infty}(G)$ given by $\Phi_{1}(\hat{\xi})=\Phi(\xi)$. For $H \in \ell^{1}(G)^{* *}=\ell^{\infty}(G)^{*}$, we define $\stackrel{\vee}{H} \in \ell^{1}(S)^{* *}$ by $\stackrel{\vee}{H}(\xi)=H(\hat{\xi})$. Then $(\Phi)^{\wedge \vee}=\Phi$ for $\Phi \in \ell^{1}(S)^{* *}$. Next, for $\xi \in \ell^{\infty}(S), a \in G$, $b=\pi(a)$, we have $\ell_{a} \hat{\xi}=\left(\ell_{b} \xi\right)^{\wedge}$ and, therefore, for $H \in \ell^{1}(G)^{* *}$,

$$
(H \hat{\xi})(a)=H\left(\ell_{a} \hat{\xi}\right)=H\left(\left(\ell_{b} \xi\right)^{\wedge}\right)=\stackrel{\vee}{H}\left(\ell_{b} \xi\right)=(\stackrel{\vee}{H} \xi)(b)=(\stackrel{\vee}{H} \xi)^{\wedge}(a)
$$

So, $H \hat{\xi}=(\stackrel{\vee}{H \xi})^{\wedge}$ and further, $H \hat{\xi}$ is in $\ell_{\pi}^{\infty}(G)$. Moreover,

$$
\stackrel{\vee}{M}\left(\mathbf{1}_{s}\right)=M(\mathbf{1})=1=\|M\|=\|\stackrel{\vee}{M}\| \text {. }
$$

So $\stackrel{\vee}{M}$ is a mean on $S$. Now let $\stackrel{\vee}{\mathcal{F}}=\{\stackrel{\vee}{F}: F \in \mathcal{F}\}$. Let $F \in \mathcal{F}$ and $\Phi \in \ell^{1}(S)^{* *}$. Put $H=\hat{\Phi}$. Since $\mathcal{F}$ is an invoid for $G$ there exist $F_{j} \in \mathcal{F}, \beta_{j} \in \mathbb{C}, 1 \leq j \leq n$ satisfying

$$
H \square F=\sum_{j=1}^{n} \beta_{j} F_{j} .
$$


So for $\xi \in \ell^{\infty}(S)$,

$$
H(\hat{F \xi})=\sum_{j=1}^{n} \beta_{j} F_{j}(\hat{\xi})
$$

which in turn gives that

$$
H\left((\stackrel{\vee}{F} \xi)^{\wedge}\right)=\sum_{j=1}^{n} \beta_{j}\left(F_{j}\right)^{\vee}(\xi)
$$

i.e.

$$
\stackrel{\vee}{H}(\stackrel{\vee}{F} \xi)=\left(\sum_{j=1}^{n} \beta_{j}\left(F_{j}\right)^{\vee}\right)(\xi)
$$

So

$$
\Phi(\stackrel{\vee}{F} \xi)=\left(\sum_{j=1}^{n} \beta_{j}\left(F_{j}\right)^{\vee}\right)(\xi)
$$

Therefore,

$$
\Phi \square \stackrel{\vee}{F}=\sum_{j=1}^{n} \beta_{j}\left(F_{j}\right)^{\vee} .
$$

Thus $\mathcal{F}$ is an invoid for $S$.

As $\stackrel{\vee}{M} \in \stackrel{\vee}{\mathcal{F}}$ we conclude that $\stackrel{\vee}{\mathcal{F}}$ is a meanoid for $S$. The result follows on noting that $\# \stackrel{\vee}{\mathcal{F}} \leq \alpha$

The second part follows from the first just by applying it to the canonical homomorphism of $G$ onto $G / N$.

(ii) Let $G / S$ be the set of left cosets $[x]=x S$. Let $h$ be any function on $G / S$ to $G$ with $h([x]) \in[x]$ for each $[x]$ in $G / S$ and $h([e])=e$, the identity of $G$. Let $C$ be the range of $h$. Then $h_{0}=\chi_{C}$ is a Bruhat function attached to the pair $(G, S)$. For $\xi \in \ell^{\infty}(S)$, let $\hat{\xi}(x)=\xi\left(x h\left[x^{-1}\right]\right)$ for $x$ in $G$. Then $\hat{\xi} \in \ell^{\infty}(G)$. Also for $a \in S, \hat{\xi}(a)=$ $\xi\left(a h\left(\left[a^{-1}\right]\right)\right)=\underbrace{}_{\vee}(a e)=\xi(a)$. So $\hat{\xi} \mid S=\xi$. For $H \in \ell^{1}(G)^{* *}$, let $\stackrel{\vee}{H} \in \ell^{1}(S)^{* *}=\ell^{\infty}(S)^{*}$ be defined by $\stackrel{\vee}{H}(\xi)=H(\hat{\xi})$. Consider any $H \in \ell^{1}(G)^{* *}, \xi \in \ell^{\infty}(S)$ and $a \in S$. Then for $x \in G,\left(\ell_{a} \xi\right)^{\wedge}(x)=\ell_{a}\left(x h\left(\left[x^{-1}\right]\right)\right)=\xi\left(\operatorname{axh}\left(\left[(a x)^{-1}\right]\right)\right)=\hat{\xi}(a x)=\ell_{a} \hat{\xi}(x)$; and, therefore, $\left(\ell_{a} \xi\right)^{\wedge}=\ell_{a} \hat{\xi}$. So

$$
(H \hat{\xi})(a)=H\left(\ell_{a} \hat{\xi}\right)=H\left(\left(\ell_{a} \xi\right)^{\wedge}\right)=\stackrel{\vee}{H}\left(\ell_{a} \xi\right)=\stackrel{\vee}{H} \xi(a)
$$


So $\stackrel{\vee}{H} \xi=(H \hat{\xi}) \mid S$. For $\Phi \in \ell^{1}(S)^{* *}$, let $\stackrel{\wedge}{\Phi}: \ell^{\infty}(G) \rightarrow \mathbb{C}$ be given by $\hat{\Phi}(f)=\Phi(f \mid S)$. Then $\hat{\Phi} \in \ell^{\infty}(G)^{*}=\ell^{1}(G)^{* *}$. Also

$$
(\hat{\Phi})^{\vee}(\xi)=\hat{\Phi}(\hat{\xi})=\Phi(\hat{\xi} \mid S)=\Phi(\xi)
$$

So $(\hat{\Phi})^{\vee}=\Phi$.

Let $\mathcal{F}$ be a meanoid for $G$ with $\# \mathcal{F} \leq \alpha$ and $M$ a mean present in $\mathcal{F}$. Let $F \in \mathcal{F}$ and $\Phi \in \ell^{1}(S)^{* *}$. Since $\mathcal{F}$ is a meanoid, there exist $F_{j} \in \mathcal{F}, \beta_{j} \in \mathbb{C}, 1 \leq j \leq n$ such that $\hat{\Phi} \square F=\sum_{j=1}^{n} \beta_{j} F_{j}$. So for $\xi \in \ell^{\infty}(S)$,

$$
\hat{\Phi}(\hat{F \xi})=\sum_{j=1}^{n} \beta_{j} F_{j}(\hat{\xi})
$$

i.e.,

$$
\Phi(F \hat{\xi} \mid S)=\sum_{j=1}^{n} \beta_{j} \stackrel{\vee}{F}_{j}(\xi)
$$

which in turn, gives

$$
\Phi(\stackrel{\vee}{F} \xi)=\sum_{j=1}^{n} \beta_{j} \stackrel{\vee}{F}_{j}(\xi)
$$

So

$$
\Phi \square \stackrel{\vee}{F}=\sum_{j=1}^{n} \beta_{j} \stackrel{\vee}{F}_{j} .
$$

Thus $\stackrel{\vee}{\mathcal{F}}=\{\stackrel{\vee}{F}: F \in \mathcal{F}\}$ is an invoid for $S$. Also $\stackrel{\vee}{M}\left(\mathbf{1}_{s}\right)=M\left(\hat{\mathbf{1}}_{s}\right)=M(\mathbf{1})=1$. Therefore, $\stackrel{\vee}{\mathcal{F}}$ is a meanoid for $S$ and $\# \stackrel{\vee}{\mathcal{F}} \leq \alpha$. Hence $S$ is $\alpha$-amenable.

(iii) Let $M$ be a left invariant mean on $N, \mathcal{F}$ a meanoid for $G / N$ with $\# \mathcal{F} \leq \alpha$ and $\Phi$ a mean present in $\mathcal{F}$. Consider any $f \in \ell^{\infty}(G)$. For $x \in G$, the function $f_{1}^{(x)}=\ell_{x} f$ restricted to $N$ is in $\ell^{\infty}(N)$. Also for $a \in N, \ell_{a} f_{1}^{(x)}=f_{1}^{(x a)}$. We define $\widetilde{f} \in \ell^{\infty}(G)$ by $\tilde{f}(x)=M\left(f_{1}^{(x)}\right)$ for $x$ in $G$. Then, for $x \in G$ and $a \in N, \widetilde{f}(x a)=M\left(f_{1}^{(x a)}\right)=$ $M\left(\ell_{a} f_{1}^{(x)}\right)=M\left(f_{1}^{(x)}\right)=\widetilde{f}(x)$. So $\widetilde{f}$ is constant on the left coset $x N$. Thus we may define $\hat{f} \in \ell^{\infty}(G / N)$ by putting $\hat{f}(x N)=\tilde{f}(x)$.

For $x, y \in G$ and $f \in \ell^{\infty}(G),\left(\ell_{y} f\right)^{\wedge}(x N)=\left(\ell_{y} f\right)^{\sim}(x)=M\left(\left(\ell_{y} f\right)_{1}^{(x)}\right)=M\left(\ell_{y x} f\right)=$ $M\left(f_{1}^{(y x)}\right)=\widetilde{f}(y x)=\hat{f}(y x N)=\hat{f}((y N)(x N))=\ell_{y N} \hat{f}(x N)$. 
Now for $\Psi \in \ell^{1}(G / N)^{* *}$, let $\stackrel{\vee}{\Psi} \in \ell^{1}(G)^{* *}=\ell_{\infty}(G)^{*}$ be given by $\stackrel{\vee}{\Psi}(f)=\Psi(\hat{f})$. Then for $f \in \ell^{\infty}(G), y \in G$ we have

$$
\begin{aligned}
\stackrel{\vee}{\Psi} f(y) & =\stackrel{\vee}{\Psi}\left(\ell_{y} f\right)=\Psi\left(\left(\ell_{y} f\right)^{\wedge}\right)=\Psi\left(\ell_{y N} \hat{f}\right) \\
& =(\Psi \hat{f})(y N) .
\end{aligned}
$$

Thus, $\stackrel{\vee}{\Psi} f$ is constant on each left $\operatorname{coset} x N$ for $x \in G$. Let $Y=\left\{f \in \ell^{\infty}(G / N): f\right.$ is constant on left cosets of $N\}=\left\{\xi \circ \pi: \xi \in \ell^{\infty}(G / N)\right\}$, where $\pi$ is the canonical map given by $\pi(x)=x N$. Then $\ell^{\infty}(G / N)$ can be identified with $Y$ by $\xi \rightarrow \hat{\xi}=\xi \circ \pi$. For $H \in \ell^{1}(G)^{* *}$ let $\hat{H} \in \ell^{1}(G / N)^{* *}=\ell^{\infty}(G / N)^{*}$ be given by $\hat{H}(\xi)=H(\hat{\xi})$.

Let $\stackrel{\vee}{\mathcal{F}}=\{\stackrel{\vee}{\Psi}: \Psi \in \mathcal{F}\}$. Then for $\Psi \in \mathcal{F}, H \in \ell^{1}(G)^{* *}$, there exist $\Psi_{j} \in \mathcal{F}, \beta_{j} \in \mathbb{C}$, $1 \leq j \leq n$ with $\hat{H} \square \Psi=\sum_{j=1}^{n} \beta_{j} \Psi_{j}$

Now for $f \in \ell^{\infty}(G)$,

$$
\begin{aligned}
(H \square \stackrel{\vee}{\Psi})(f) & =H(\stackrel{\vee}{\Psi})=H\left((\Psi \hat{f})^{\wedge}\right)=\hat{H}(\Psi \hat{f}) \\
& =(\hat{H} \square \Psi)(\hat{f})=\left(\sum_{j=1}^{n} \beta_{j} \Psi_{j}\right)(\hat{f})=\sum_{j=1}^{n} \beta_{j} \Psi_{j}(\hat{f}) \\
& =\sum_{j=1}^{n} \beta_{j}\left(\Psi_{j}\right)^{\vee}(f) .
\end{aligned}
$$

So $H \square \stackrel{\vee}{\Psi}=\sum_{j=1}^{n} \beta_{j}\left(\Psi_{j}\right)^{\vee}$.

Therefore, $\stackrel{\vee}{\mathcal{F}}$ is an invoid for $G$. Also $\stackrel{\vee}{\Phi}$ is a mean on $G$ and as a consequence, $\stackrel{\vee}{\mathcal{F}}$ is a meanoid for $G$. As $\# \stackrel{\vee}{\mathcal{F}} \leq \# \mathcal{F} \leq \alpha$ we have the desired result.

Corollary 2.8. Let $G$ be an infinite discrete group with $\# G=k$.

(i) Let $G$ be subamenable. Let $S$ be a discrete group with $\# S=k$. If $S$ is a homomorphic image of $G$ (equivalently, a quotient group of $G$ by a normal subgroup $N$ ) or a subgroup of $G$, then $S$ is subamenable.

(ii) Let $N$ be an amenable normal subgroup of $G$ such that $G / N$ is subamenable. Then $G$ is subamenable. In particular, it is so if $N$ is taken to be $G_{F C}$, the subgroup of $G$ consisting of elements in $G$ with finite conjugacy classes. 
Proof. We have only to note that all through subamenability of $G$ or $S$ can be replaced by $\alpha$-amenability for some $\alpha<2^{2^{k}}$ and then apply Proposition 2.7 above. For the second part of (ii) we use the well-known fact that $G_{F C}$ is amenable (cf. [Pi, Proposition 12.9]).

Theorem 2.9. (i) Every discrete group $G$ is a subgroup of a subamenable discrete group $G_{\sigma}$ with $\# G_{\sigma} \leq 2^{\# G}$.

(ii) There exist non-amenable subamenable groups of any infinite cardinality $\geq c$, the cardinality of the continuum.

(iii) Every discrete group $G$ is a subgroup of a non-amenable subamenable discrete group $G_{\Sigma}$ with $\# G_{\Sigma} \leq 2^{\# G \alpha_{0}}$.

Here $\alpha_{0}$ is the cardinality of the set $\mathbb{N}$ of natural numbers.

Proof. (i) Let $G$ be any discrete group. If $G$ is finite then it is amenable (and, therefore, subamenable). Now let $G$ be infinite with $k=\# G \geq \alpha_{0}$. Then by Remark 2.3(v), $G$ is $\alpha$-amenable for some $\alpha \leq 2^{2^{k}}$. Let $\ell>k$ be any cardinal number. Let $N$ be any discrete amenable group with $\# N=\ell$. We may take $N$ to be any abelian group with cardinality $\ell$, the free abelian group with $\ell$ generators, for instance. Let $G_{\ell}$ be the product group $N \times G$. By Proposition 2.7(iii) above, $G_{\ell}$ is $\alpha$-amenable. Now $k_{\ell}=\# G_{\ell}=\ell . k=\ell>k$. So $2^{2^{k_{\ell}}}=2^{2^{\ell}}>2^{2^{k}} \geq \alpha$. So $G_{\ell}$ is subamenable. We put $G_{\sigma}=G_{\ell}$ with $\ell=2^{k}=2^{\# G}$.

(ii) We take $G$ to be any non-amenable countable group, say the free group $\mathbb{F}_{2}$ with two generators and then take $\ell$ to be any infinite cardinal number $\geq c$ in the proof of (i) above. Then $G_{\ell}$ is not amenable simply because it has a non-amenable subgroup $G$, whereas every subgroup of an amenable discrete group is amenable.

(iii) Given any group $G$, we let $\Gamma=G \times \mathbb{F}_{2}$ and then apply part(i) to $\Gamma$ (in place of $G)$ and put $G_{\Sigma}=\Gamma_{\sigma}$.

Remark 2.10. (i) Obviously, in Theorem 2.9(i) above if $G$ is subamenable we may take $G_{\sigma}=G$; but we have divided the proof of Theorem 2.9(i) above into easily checkable cases $G$ finite or $G$ infinite rather than $G$ subamenable and $G$ nonsubamenable. If $G$ is a non-subamenable group and $G_{1}$ is a group containing $G$ as a subgroup then, in view of Corollary 2.8(i), $\# G_{1}=\# G$ forces $G_{1}$ to be nonsubamenable, and, therefore if $G_{1}$ is a subamenable group we must have $\# G_{1}>\# G$. In the statement and the last step (sentence) of the proof of Theorem 2.9(i) we have used cardinal number $2^{\# G}$ to avoid any questions regarding the truth of generalized continuum hypothesis $(\mathrm{GCH})$ or otherwise. 
(ii) Theorem 2.9(i) has no amenable counterpart because of the well-known fact that a subgroup of an amenable discrete group is amenable (already used in the proof of part (ii) of Theorem 2.9 above) and the fact that there exist non-amenable groups.

(iii) On the opposite side, if $G$ has an element $x$ of infinite order then it contains the infinite cyclic group generated by $x$, which is abelian and, therefore, amenable; and, on the other hand, if $G$ is a torsion group then also it contains a finite (and, therefore) amenable subgroup.

(iv) There is a vast standard literature on torsion groups (and other groups) vis-à-vis amenability created by mathematicians such as S.I. Adian, R.I. Grigorchuk, A.Y. Ol'shanskii, D. Osin, M.V. Sapir and J. Jits.

We have yet to properly utilize it for our purposes.

Remark 2.11. (i) Let us call a group $G$ Arens-* if $\ell^{1}(G)^{* *}$ has an involution. Every finite group is Arens- $*$ and we have not been able to give an example of an Arens-* infinite group.

(ii) Theorem 2.2, and Theorem 2.9 put together give that

(a) every group $G$ is a subgroup of some non Arens-* group $G_{\sigma}$ with $\# G_{\sigma} \leq 2^{\# G}$,

(b) there are non-amenable non-Arens-* groups of all cardinal numbers $\geq c$,

(c) every infinite amenable group is non-Arens-* and is a subgroup of a nonamenable non-Arens-* group,

(d) every group $G$ is a subgroup of a non-amenable non-Arens-* group $G_{\Sigma}$ with $\# G_{\Sigma} \leq 2^{\# G \alpha_{0}}$.

(iii) Every non-torsion group $G$ is sandwiched between some pair of non-Arens-* groups. To see this we have only to combine (ii)(a) above with Remarks 2.10(iii) above. In fact, there are infinitely many such pairs in view of the fact that every infinite cyclic group has infinitely many infinite cyclic subgroups and on the other hand, (ii)(a) above indicates how to build up a chain of non-Arens-* groups containing $G$ as a subgroup.

(iv) An infinite torsion group $G$ is sandwiched between some pair consisting of a finite Arens-* group and a non-Arens-* group. Again, there are infinitely many such pairs.

Remark 2.12. (i) It is too well-known (see [CY], [Do], [DLS] for instance) that $\ell^{1}(G)^{* *}$ can be identified with the space of finitely additive measures on $G$ or, equivalently, with the space $M(\beta G)$ of complex regular Borel measures on the Stone-Cech compactification $\beta G$ of $G$. Further, under the Arens product $\square$ of $\ell^{1}(G)^{* *}, \beta G$ (identified with unit point masses) is a compact right topological semigroup equipped with the weak*-topology coming from $\ell^{\infty}(G)$. 
(ii) [DLS], Proposition 7.5(i) gives that if $L$ is a closed left ideal in $\beta G$ then $M(L)$ is a weak*-closed left ideal in $M(\beta G)$.

(iii) There is an abundance of closed left ideals in $\beta G$, this follows as a special case of discrete $G$ from Proposition 2 of Lau and A. L. T. Paterson [LPa]. Keeping the proof of this Proposition, particularly the proof of Lemma 3, in mind we record it as: If $G$ is infinite discrete group, $\beta G$ contains at least $2^{2^{\# G}}$ minimal closed left ideals which are mutually disjoint.

(iv) A combination of (ii) and (iii) above gives that $M(\beta G)$ contains at least $2^{2^{\# G}}$ weak*-closed left ideals whose pairwise intersetion is $\{0\}$. Hence, if $M(\beta G)$ has an involution then it must have at least $2^{2^{\# G}}$ right ideals whose pairwise intersetion is $\{0\}$. We do not know the situation in this regard.

(v) In the absence of involution on $M(\beta G)$ for many groups (as seen above), we may consider involutions on subalgebras of $\ell^{1}(G)^{* *}$. The algebra $\ell^{1}(G)$ and its (algebraic) centre (which, in fact, turns out to be the centre of $\ell^{1}(G)^{* *}$ as well (using [L], [LL] and is, therefore, invariant under an involution, if any, on $\left.\ell^{1}(G)^{* *}\right)$ have their own involutions (see [Pat]). There is also an abundance of such subalgebras of $\ell^{1}(G)^{* *}$ which do not intersect $\ell^{1}(G)$ except for $\{0\}$. In fact, apart from the group $G$, the semigroup $\beta G$ very often contains several groups (considered as subsemigroups of $\beta G$ ) contained in $\beta G \backslash G$. T. Budak, N. Isik and J. Pym [BIP], N. Hindman and D. Strauss ([HS2], [HS4]), I. Protasov $[\mathrm{Pr}]$ ) and $\mathrm{Yu}$ Zelenyuk [Z], for instance, give concrete examples and some general results (see also $[\mathrm{DLS}])$.

(vi)This brings us to the question of involutions on subalgebras of $L^{1}(G)^{* *}$ for a locally compact group $G$ as well (see [FG], [LPy], [S]).

(vii) Another idea is to consider a trivolution on $L^{1}(G)^{* *}$ viz., a conjugate-linear antihomomorphism $\rho$ satisfying $\rho^{3}=\rho$. It is as if $\rho$ is its own generalized inverse instead of being inverse. The name is motivated by a notion studied by J.W. Degen [De]. One can find details in [FMS].

\section{ACKNOWLEDGEMENTS}

The author would like to thank Mahmoud Filali, Kenneth A. Ross and K.R. Parthasarathy for useful discussion from time to time. She also thanks H. Farhadi and F. Ghahramani for their useful comments related to the topic.

The author thanks the referee for his useful suggestions, particularly that of including Remark 2.12 (iii). This prompted her to add items (ii) and (iv) also in Remark 2.12. 
She thanks Indian National Science Academy for support under the INSA Senior Scientist Programme and Indian Statistical Institute, New Delhi for a Visiting Professorship under this programme together with excellent research facilities.

\section{REFERENCES}

[A1] Richard Arens: Operations induced in function classes, Monatsh. für Math., 55 (1951), 1-19.

[A2] Richard Arens: The adjoint of a bilinear operation, Proc. Amer. Math. Soc., 2 (1951), 839-848.

[BIP] Talin Budak, Nilgun Isik and John Pym: Subsemigroups of Stone-Čech compactifications, Math. Proc. Camb. Phil. Soc. 116 (1994), 99-118.

[CY] Paul Civin and Bertram Yood: The second conjugate space of a Banach algebra as an algebra, Pacific J. Math., 11 (2) (1961), 847-870.

[DLS] H. G. Dales, A. T.-M. Lau and D. Strauss: Banach algebras on semigroups and on their compactifications, Mem. Amer. Math. Soc. 205 (2010), No. 966, vi+165 pp.

[Da] M.M. Day: Amenable semigroups, Illinois J. Math., 1 (1957), 509-544.

[De] J. W. Degen: Some aspects and examples of infinity notions, Math. Logic Quart., 40 (1) (1994), 111-124.

[Do] R. G. Douglas: Generalized group algebras, Illinois J. Math., 10 (1966), 309-321.

[DH] J. Duncan and S.A.R. Hosseiniun: The second dual of a Banach algebra, Proc. Royal Soc. Edinburgh, Section A, 84 (1979), 309-325.

[FG] H. Farhadi and F. Ghahramani: Involutions on the second duals of group algebras and a multiplier problem, Proc. Edinb. Math. Soc., 50 (2)(2007), No. 1, 153-161.

[F1] M. Filali: Finite-dimensional right ideals in some algebras associated with a locally compact group, Proc. Amer. Math. Soc., 127 (1999), 1729-1734.

[F2] M. Filali: Finite-dimensional left ideals in some algebras associated with a locally compact group, Proc. Amer. Math. Soc., 127 (1999), 2325-2333.

[FMS] M. Filali, M. Sangani Monfared, Ajit Iqbal Singh: Ideals and involutions in algebras related to the second duals of group algebras, Preprint.

[FP] M. Filali and J.S. Pym: Right cancellation in the $\mathcal{L U C}$-compactification of a locally compact group, Bull. London Math. Soc., 35 (2003), 128-134.

[FSa] Mahmoud Filali and Pekka Salmi: One-sided ideals and right cancellation in the second dual of the group algebra and similar algebras, J. London Math. Soc., 75(2) (2007), 35-46. 
[FSi] Mahmoud Filali and Ajit Iqbal Singh: Recent developments on Arens regularity and ideal structure of the second dual of a group algebra and some related topological algebras, General topological algebras (Tartu, 1999), 95-124, Math. Stud. (Tartu) 1, Est. Math. Soc., Tartu, 2001.

[G] Michael Grosser: Algebra involutions on the bidual of a Banach algebra, Manuscripta Math., 48 (1-3) (1984), 291-295.

[HR] Edwin Hewitt and Kenneth A. Ross: Abstract Harmonic Analysis, Vol. 1 and Vol. 2, Springer Verlag, New York, Berlin, 1963 (2nd edition 1979) and 1970.

[H] Neil Hindman: Algebra in $\beta S$ and its applications to Ramsey theory, Math. Japonica, 44 (3) (1996), 581-625.

[HS1] N. Hindman and D. Strauss, Algebra in the Stone-Čech compactification: Theory and Applications, de Gruyter, Berlin, 1998.

[HS2] N. Hindman and D. Strauss, Discrete groups in $\beta \mathbb{N}$, Topology Appl., 154 (2007), 2099-2103.

[HS3] N. Hindman and D. Strauss: Algebra in the space of ultrafilters and Ramsey theory, Contemporary Mathematics, 530 (2010), 121-145.

[HS4] N. Hindman and D. Strauss: More discrete copies of $\mathbb{Z}$ in $\beta \mathbb{N}$, Topology Appl., 157 (2010), 2145-2156.

[L] A.T.-M. Lau: Continuity of Arens multiplication on the dual space of bounded uniformly continuous functions on locally compact groups and topological semigroups, Math. Proc. Camb. Phil. Soc., 99 (1986), 53-63.

[LL] A.T.-M. Lau and V. Losert: On the second conjugate algebra of $L_{1}(G)$ of a locally compact group, J. London Math. Soc., 37 (2) (1988), 464-470.

[LPa] Anthony To-Ming Lau and Alan L. T. Paterson: The exact cardinality of the set of topological left invariant means on an amenable locally compact group,Proc. Math. Soc., 98 (1986), No.1, 75-80.

[LPy] Anthony To-Ming Lau and John Pym: Concerning the second dual of the group algebra of a locally compact group, J. London Math. Soc., 41 (2)(1990), 445-466.

[N] Matthias Neufang: Solution to Farhadi-Ghahramani's multiplier problem, Proc. Amer. Math. Soc., 138 (2) (2010), 553-555.

[Pal] Theodore W. Palmer: Banach Algebras. Vol. 1 and Vol. 2, Encylopedia of Mathematics and its Applications, 49 and 79, Cambridge University Press, 1994 and 2001.

[Pat] Paul L. Patterson: Involutions on algebras arising from locally compact groups, Proc. Amer. Math. Soc., 121 (3) (1994), 739-745. 
[Pi] J.P. Pier: Amenable Locally Compact Groups, John Wiley \& Sons, 1984.

[Pr] I. Protasov: Finite groups in $\beta G$, Mat. Stud., 10 (1998), no.1, 17-22.

[S] Ajit Iqbal Singh: $L_{0}^{\infty}(G)^{*}$ as the second dual of the group algebra $L^{1}(G)$ with a locally convex topology, Michigan Math. J., 46 (1999), 143-150.

[Y] N. J. Young: The irregularity of multiplication in group algebras, Quart. J. Math. Oxford, Ser. (2) 24 (1973), 59-62.

[Z] Y. Zelenyuk: Finite groups in $\beta \mathbb{N}$ are trivial, Semigroup Forum, 55 (1997), 131-132.

Theoretical Statistics and Mathematics Unit, Indian Statistical Institute, 7, S. J. S. Sansanwal Marg, New Delhi-110 016, India

E-mail address: aisingh@isid.ac.in, aisingh@sify.com 